\title{
Urbanes Sedimentmanagement - vom Einzugsgebiet bis zum Kanal
}

\author{
Johannes Schobesberger - Thilo Lehmann · Andreas Kimmersdorfer • Petr Lichtneger • Christine Sindelar • \\ Helmut Habersack
}

Online publiziert: 8. Januar 2019

(c) Der/die Autor(en) 2019

Zusammenfassung Sedimente spielen im urbanen Gebiet sowie in der Kanalisation eine bedeutende Rolle. Der Sedimenttransport stellt dabei einen signifikanten Risiko- und Kostenfaktor dar, der häufig Handarbeit zur Beseitigung von Anlandungen erfordert. Dieser Artikel behandelt ein Beispiel aus Wien (1190), welches sich aber in jedem urbanen Umfeld als Thema ergibt. Gegenstand der vorliegenden Untersuchung war es, die Sedimentherkunft im Bereich des Entlastungsbauwerks (RA 3) in der Heiligenstädter Straße zu untersuchen, da es hier zu Verlandungen im rechten Hauptsammelkanal (RHSK) kommt. Sedimenteintrag in diesem Gebiet ist über die Wienwaldbäche (z.B. Erbsenbach) und über Straßeneinläufe im urbanen Bereich möglich. Es besteht der Verdacht, dass es im Schotterfang des seitlich zufließenden Krottenbachkanals bei extremen Abflüssen $\mathrm{zu}$ Sedimentausspülungen kommt und ausgetragene Sedimente im RHSK verlanden. Dadurch wäre die hydraulische sowie sedimentologische Rückhaltewirkung des Bauwerks nicht mehr ausreichend gegeben. Die Sedimentherkunft wurde durch Sieb-

\section{J. Schobesberger $(\bowtie)$. \\ DI Dr. P. Lichtneger . \\ DI Mag. Dr. C. Sindelar \\ Univ.-Prof. DI Dr. H. Habersack \\ Department für Wasser - \\ Atmosphäre - Umwelt, Institut \\ für Wasserbau, Hydraulik und Fließgewässerforschung, Christian Doppler Labor für Sedimentforschung und -management, Universität für Bodenkultur Wien, Muthgasse 18, Wien, Österreich johannes.schobesberger@boku.ac.at}

DI T. Lehmann .

DI Dr. A. Kimmersdorfer

Fachbereich Planung, Gruppe Kanalnetzbewirtschaftung, Wien Kanal, Modecenterstraße 14c, Wien, Österreich analysen, Geröllanalysen (geologische Untersuchungen) sowie durch Geschiebemessungen (Erbsenbach) im natürlichen Bereich des Projektgebiets (Wienerwald) als auch im urbanen Bereich bestimmt. Zur Analyse der hydraulischen und sedimentologischen Rückhaltewirkung wurde der Schotterfang in einem physikalischen Modell (Maßstab 1:10) im Wasserbaulabor der Universität für Bodenkultur Wien nachgebaut. Die Ergebnisse weisen auf einen urbanen Herkunftsort der verlandeten Sedimente im RHSK hin. Bei den Geschiebemessungen konnte, trotz erhöhter Abflüsse, kaum Sedimenttransport im Erbsenbach festgestellt werden, wodurch die gute Funktion der Geschieberückhaltemaßnahmen im Einzugsgebiet bestätigt wird. Aus dem physikalischen Modellversuch ging hervor, dass die im Schotterfang verlandeten Sedimente bei erhöhten Abflüssen wahrscheinlich ausgespült werden und im RHSK verlanden. Durch die hydraulische Optimierung des Schotterfangs konnte die Sedimentrückhaltewirkung im physikalischen Modell von 18 auf $95 \%$ gesteigert werden.

\section{Schlüsselwörter}

Sedimentmanagement im Kanal Physikalisches Modell · Hydraulische Optimierung $\cdot$ Schotterfang

\section{Urban sediment management - from the catchment area to the sewer}

Abstract Sediments are important in urban areas and sewerage systems maintenance. Sediment transport is a significant risk and cost factor, which often requires manual work to remove cumulated sediments. This article deals with an environmental urban issue from a case study in the $19^{\text {th }}$ district of Vienna City. The objective of the present study was to investigate the sedimentation source in the area of the rain outlet 3 (RA 3) in Heiligenstädter
Straße as it occurs along the right main sewer (RHSK) in the sewerage system. Sediment input in this area is possible via creeks "Wienwaldbäche" (e.g. Erbsenbach) and street inlets in the urban area. In the case of extreme discharges, it is suspected that in the gravel trap of the laterally flowing sewer (Krottenbachkanal), sediments are spilled out and deposited in the RHSK. As a result, the hydraulic and sediment retention effect of the structure would no longer be sufficient. The sediment source was determined by comparing the characteristic grain sizes, the geological composition and by bedload measurements (Erbsenbach) in the natural area of the project (Wienerwald) as well as in the urban area. To analyse the hydraulic and sedimentological retention effect, the gravel trap was reconstructed in a physical model at a scale of 1:10 in the hydraulic engineering laboratory of the University of Natural Resources and Life Sciences Vienna. The results indicate an urban origin of the sediments in the RHSK. Despite increased runoff, the bedload measurements also showed hardly any sediment transport in the Erbsenbach. This confirms the good function of the bedload retention measures in the catchment area. The physical model experiment showed that the sediments deposited in the gravel trap are likely to be spilled out during increased discharge. The sediment retention effect of the gravel trap could be increased from 18 to $95 \%$ by means of installed hydraulic optimization in the physical model.

Keywords Sediment management in sewerage systems - Physical model · Hydraulic optimization - Gravel trap

\section{Einleitung}

Die einwandfreie Ableitung von Oberflächenwasser sowie von Abwasser ist eines der wesentlichsten Grundelemente urbaner Gebiete. Abwasserkanäle 
bilden dabei die Basis für gute hygienische Bedingungen und für den Überflutungsschutz der urbanen und somit dicht besiedelten Gebiete. Über den Eintrag von Oberflächenwasser in den Kanal werden Sedimente mit in das Abwassersystem gespült. Beispielsweise werden täglich ca. 18 Tonnen abgelagertes Material aus dem Wiener Kanalnetz gefördert und umweltgerecht entsorgt (Wien Kanal 2017). Auf das Jahr gesehen sind das einige tausend Tonnen. Es stecken also ein enormer Arbeitsaufwand und entsprechende Kosten dahinter. Die Arbeit erfolgt außerdem unter sehr schweren körperlichen Anstrengungen, da die Sedimente teilweise per Hand aus dem Kanal entfernt werden müssen. Daher ist es von hohem Interesse, den Materialeintrag in die Kanalisation zu reduzieren bzw. das Material an Stellen im Kanal zu befördern, an denen gut bzw. maschinell geräumt werden kann. Zu diesem Zweck gibt es u.a. Schotterfänge im Kanalnetz, die ablagerungsfähiges Material auffangen sollen. Die effektive Rückhaltewirkung dieser Schotterfänge ist daher von besonderem Interesse für Wien Kanal.

Speziell im rechten Hauptsammelkanal (RHSK) im Bereich der Heiligenstädter Straße beim Entlastungsbauwerk (RA 3) wurden erhöhte Sedimentablagerungen festgestellt, die von Hand geräumt werden müssen. An dieser Stelle mündet der Krottenbachkanal mit vorgelagertem Schotterfang in den RHSK (Abb. 1). Bei den beiden Kanälen handelt es sich um Mischwasserkanäle, in denen sowohl Regenwasser als auch Schmutzwasser gemeinsam abgeleitet werden. Die Ablagerungen im RHSK wirken sich negativ auf das Abflussverhalten im Kanal, insbesondere in den seitlichen Zubringerkanälen aus. Im Starkregenfall kann die verringerte Abfuhrkapazität zu Problemen führen bzw. im Entlastungsfall zu einem verstärkten Materialaustrag in das Gewässer. Außerdem kommt es bei Ablagerungen besonders in den wärmeren Jahreszeiten zu Faulprozessen, und dies kann schließlich zu Geruchsproblemen führen. Auch wurde die Wirkungsweise des Schotterfangs beim RA 3 infrage gestellt. Erfahrungsberichte legen nahe, dass der Schotterfang bei höheren Abflussmengen teilweise ausgespült wird. Im konkreten Fall wurde ursprünglich vermutet, dass die Sedimente zumindest teilweise aus den Wienerwaldbächen (Krottenbach und Erbsenbach) eingetragen werden, die im Wienerwald (natürliches Einzugsgebiet) frei fließen und anschließend im stärker bebauten Gebiet des 19. Wiener Bezirks (urbanes Einzugsgebiet) in die Kanalisation einmünden (Abb. 1).

Daraus ergibt sich als erste Zielsetzung eine genaue Betrachtung der Herkunft des abgelagerten Materials im Schotterfang bzw. des RHSK. Dabei ist sowohl das natürliche Einzugsgebiet mit den Wienerwaldbächen (Krottenbach und Erbsenbach) sowie das urbane Einzugsgebiet Teil der Untersuchung. Gegenstand der zweiten Zielsetzung ist die Untersuchung der hydraulischen sowie der sedimentologischen Rückhaltewirkung des Schotterfangs. Auf Basis der Gesamtbetrachtung über die Sedimentherkunft im natürlichen sowie im urbanen Einzugsgebiet und der Analyse der Verlandungssituation im Schotterfang bzw. im RHSK sollen künftig gezielte Maßnahmen an problematischen Stellen gesetzt werden, um die Verlandungen im RHSK zu reduzieren.

\section{Methodik}

Um die beiden oben genannten Zielsetzungen zu erreichen, wurden verschiedene Messungen durchgeführt. Dabei kann prinzipiell zwischen Freilandmessungen und Laboruntersuchungen unterschieden werden. $\mathrm{Zu}$ den Freilandmessungen zählen die Beprobungen des natürlichen sowie des urbanen Einzugsgebietes und die Messung des Sedimenttransportes am Erbsenbach. Die Laboranalyse beinhaltet die Untersuchung der hydraulischen sowie sedimentologischen Wirksamkeit des Schotterfanges sowie Optimierungsmaßnahmen um die Rückhaltewirkung des Schotterfanges gegebenenfalls zu verbessern.

\subsection{Beprobung des natürlichen und urbanen Einzugsgebiets}

Wie in den Zielen festgehalten, ist es wesentlich, den Herkunftsort der Sedimente, die im Schotterfang bzw. im RHSK verlanden, zu identifizieren. Deshalb wurde das Einzugsgebiet in ein natürliches Einzugsgebiet und ein urbanes Einzugsgebiet aufgeteilt und separat untersucht. Das natürliche Einzugsgebiet charakterisiert sich durch den frei fließenden Bachverlauf des Erbsenbachs im Wienerwald und dessen Zubringerbächen (z.B. Spießbach und Gspöttgraben). In diesem Bereich entstehen die relevanten Sedimente hauptsächlich durch Erosion verwitterter Gesteine durch Regenereignisse. Im Gegensatz dazu erfolgt der Eintrag der Sedimente in das Kanalsystem über das urbane Einzugsgebiet hauptsächlich über die Straßeneinläufe, z.B. durch Streusplitt, Baustellenschutt sowie Straßenabrieb. Insgesamt wurden vier Sedimentproben im natürlichen Einzugsgebiet, geologisch gesehen in der Flyschzone (Schnabel 2002), entnommen und weitere vier im urbanen Einzugsgebiet. Zusätzlich wurde sowohl der Schotterfang und der RHSK beprobt. Abb. 1 stellt das Untersuchungsgebiet im 19. Wiener Gemeindebezirk schematisch dar.

Die Probennahme im natürlichen Einzugsgebiet (Probe 1 bis 4) wurde mithilfe eines Holzrahmens $(50 \times 50 \mathrm{~cm})$ an den in Abb. 1 markierten Stellen im Verlauf des Erbsenbachs durchgeführt. Dabei wurde innerhalb des Rahmens immer die Deckschicht abgetragen und für die weitere Analyse verwendet. Im urbanen Einzugsgebiet wurden die Sedimente aus den Schlammfängen der Straßenabläufe (Probe 5 bis 8 ), entnommen (siehe Abb. 1). Dabei handelt es sich um Vertiefungen in den Straßeneinlaufschächten, in denen Sedimente bis zur Unterkante des Abflussrohres zur Kanalisation gesammelt werden können.

Folgende Analysen der entnommenen Proben wurden durchgeführt, um den Herkunftsort der Sedimente im Schotterfang sowie im RHSK zu bestimmen: (i) Siebanalyse mit Siebmaschine (Haver-UWL 400N) zur Bestimmung der charakteristischen Korngrößen; (ii) Geröllanalysen/petrografische Beschreibungen gemäß EN 932-3 zur Bestimmung der geologischen Zusammensetzung der Proben. Mithilfe der Siebanalyse können die charakteristischen Korngrößen zwischen den verschiedenen Probestellen verglichen werden. Dies allein ist jedoch nicht ausreichend, um den Herkunftsort der Sedimente zu analysieren. Deshalb ist die Geröllanalyse wesentlich, um die geologische Zusammensetzung der Sedimentproben zu bestimmen. Das natürliche Einzugsgebiet befindet sich in der Flyschzone, weshalb hier von Gesteinen wie zum Bespiel Sandstein, Mergel und Schieferton ausgegangen werden kann (Schnabel 2002). Im urbanen Einzugsgebiet (Straßen, Gebäude usw.) ist mit einer anderen geologischen Zusammensetzung der Sedimente zu 


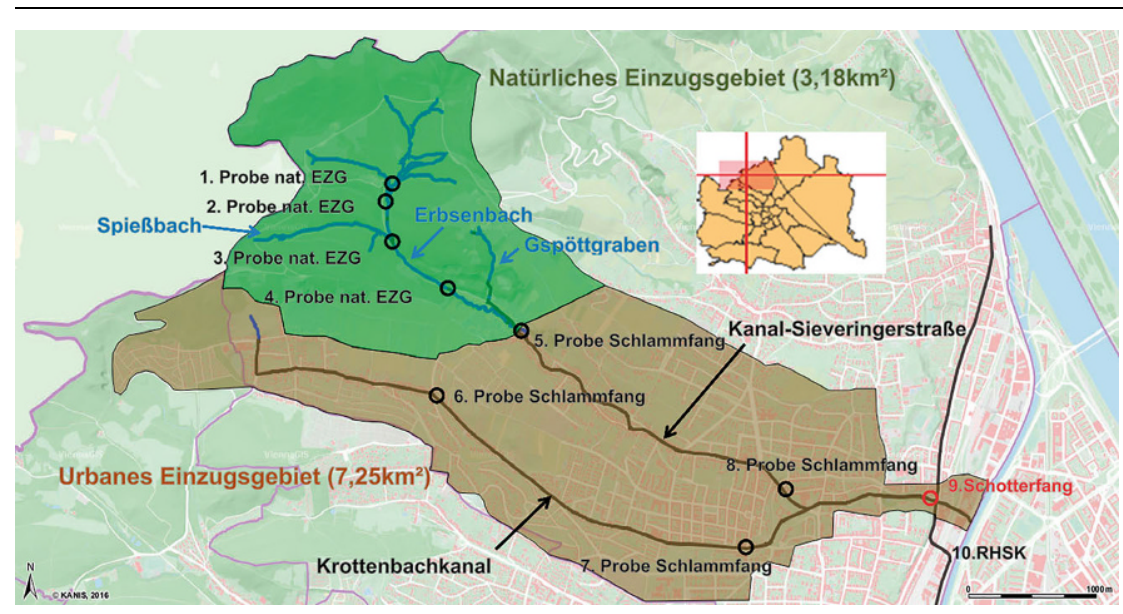

Abb. 1 SchematischeDarstellung desnatürlichen sowiedesurbanenEinzugsgebiets, der relevanten Bach-und Kanalverläufe und des Schotterfangs (Kartengrundlage: Wien Kanal-Kanis 2016)

rechnen, die beispielsweise jenen von Baumaterialien bzw. Streusplit ähnelt.

\subsection{Messungen des}

Sedimenttransports an

ausgewählten Wienerwald-Bächen (Erbsenbach)

Bevor der Erbsenbach in den Kanal in der Sieveringer Straße mündet, fließt dieser auf einer Strecke von ca. $2,3 \mathrm{~km}$ frei mit einem mittleren jährlichen Abfluss von $\mathrm{MQ}=0,04 \mathrm{~m}^{3} / \mathrm{s}$ (Abb. 1). Das einjährliche Hochwasser $\mathrm{HQ}_{1}$ entspricht einem Durchfluss von $4,0 \mathrm{~m}^{3} / \mathrm{s}$ und das hundertjährliche Hochwasser $\mathrm{HQ}_{100}$ weist einen Durchfluss von $30,0 \mathrm{~m}^{3} / \mathrm{s}$ auf. Auf einer Fließstrecke von circa $680 \mathrm{~m}$ ist der Erbsenbach als naturnah (Morphologieklasse 2) zu kategorisieren. Der weitere, flussab gelegene Fließabschnitt mit einer Länge von $1600 \mathrm{~m}$ ist als naturfern (Morphologieklasse 4) $\mathrm{zu}$ beschreiben und ist mit Rückhaltebecken sowie mit etlichen Quer- und Längsbauwerken hart verbaut (Digitale Gewässerkartei 2014).

Um den möglichen Sedimenteintrag aus dem Erbsenbach und dessen Zubringerbächen in die Kanalisation genauer in den Schotterfang bzw. in den RHSK - zu bestimmen, wurden verschiedene Methoden zur Messung des Sedimenttransports verwendet:

1. Handsammler mit einer Öffnungsweite von Länge $\mathrm{zu}$ Breite mit
$25 \times 25 \mathrm{~cm}$. Dieser Sammler wurde vor allem bei geringen Abflüssen verwendet.

2. Helley-Smith-Sampler mit einer Öffnungsweite von Länge zu Breite mit $15,2 \times 15,2 \mathrm{~cm}$. Dieser Sammler wurde bei mittleren bis hohen Abflüssen verwendet.

3. Fixer Geschiebeauffangkorb am Absturz des Erbsenbaches in die Kanalisation mit einer Öffnungsweite von Länge zu Breite mit $50 \times 50 \mathrm{~cm}$.

Mit den Sammlern 1 und 2 wurde $20 \mathrm{~m}$ flussauf der Einmündung des Erbsenbachs in den Kanal bei Regenereignissen gemessen, während Sammler 3 fix am Absturz vom Erbsenbach in die Kanalisation angebracht war, um eine Langzeitgeschiebemessung zu gewährleisten. Insgesamt wurden acht $\mathrm{Ge}$ schiebemessungen bei verschiedenen Durchflüssen durchgeführt. Parallel da$\mathrm{zu}$ wurde der Wasserstand an einem zuvor eingemessenen Profil mit Pegel aufgenommen und der Durchfluss unter Verwendung der Strickler-Formel mit einem Stricklerwert von $45 \mathrm{~m}^{1 / 3} \mathrm{~s}^{-1}$ berechnet.

\subsection{Schotterfang im physikalischen Modell und erste Optimierungen}

Der zu untersuchende Schotterfang (Abb. 2) befindet sich am östlichen Ende des Wertheimsteinparks unterhalb der Heiligenstädter Straße (1190 Wien) und ist dem RHSK vorgeschaltet, um die
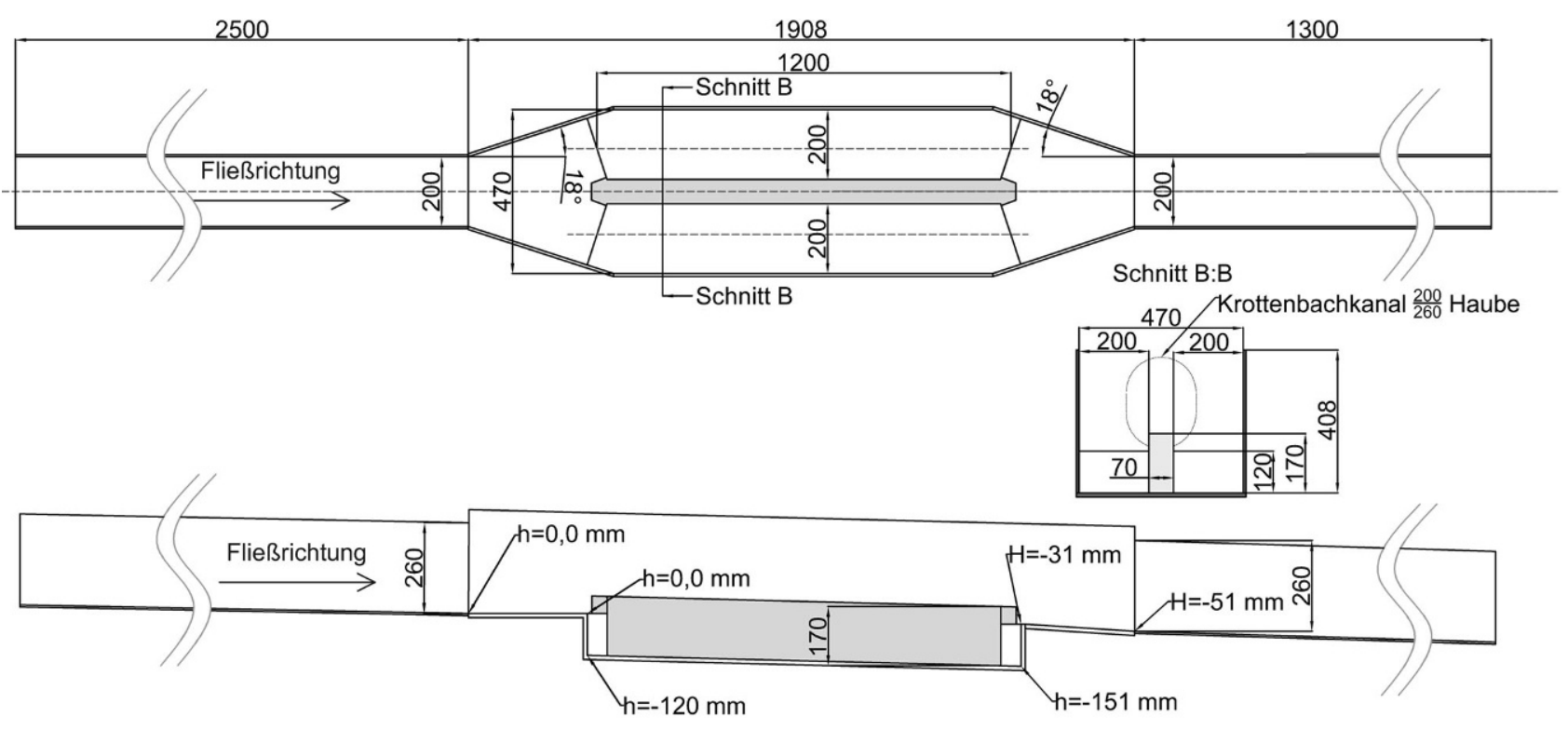

Abb. 2 Schotterfanggeometrie im Modell im Maßstab 1:10 (Modellmaße in mm) 


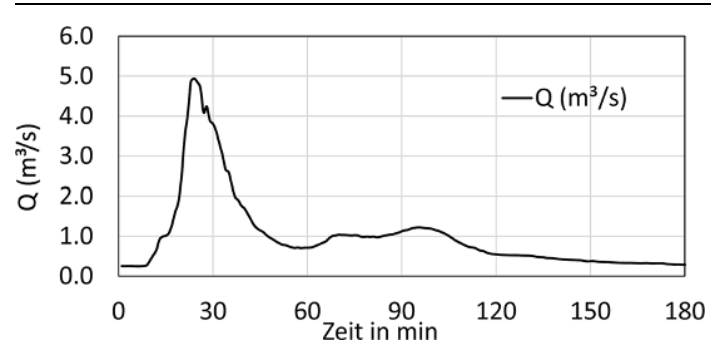

Abb. 3 Abflussganglinie im Krottenbachkanal bei erhöhtem Abfluss (Naturwerte)

Sedimente am Weitertransport zu hindern (Abb. 1). Geometrisch betrachtet wird der Schotterfang durch einen Steg in zwei Absetzbecken geteilt, mit jeweils einer Länge - Breite - Höhe von 12 - 2 $1,2 \mathrm{~m}$ in der Natur. Damit ergibt sich ein maximales Absetzvolumen von $57,6 \mathrm{~m}^{3}$. Der Übergang zwischen dem Krottenbachkanal (Haubenprofil $260 / 200 \mathrm{~cm}$ ) und dem Schotterfang besteht aus einer Aufweitung mit einem Öffnungswinkel von ca. $36^{\circ}$. Am Ende des Schotterfangs befindet sich eine Einschnürung mit demselben Winkel der Aufweitung. Der Schotterfang ist nahezu im rechten Winkel an den RHSK angeordnet. Um die hydraulische Wirksamkeit sowie die sedimentologische Rückhalte- und Absetzwirkung des Schotterfangs zu untersuchen, wurde der Schotterfang im Maßstab 1:10 im Wasserbaulabor der Universität für Bodenkultur nachgebaut und in den bestehenden Wasserkreislauf integriert. Die Abmessungen aus der Natur wurden unter Einhaltung der geometrischen Ähnlichkeit auf das Modell übertragen. Das Modell besteht aus einem Zulauf, dem Korpus des Schotterfangs und einem Ablaufbereich. Um einen beruhigten Zulauf des Wassers ins Modell zu garantieren, dienen zwei miteinander kommunizierende Einlaufbecken mit einem Volumen von $4 \mathrm{~m}^{3}$. In eines der Einlaufbecken mündet eine Rohrleitung, die an eine Wasserentnahmestation des Labors gekoppelt ist. Über eine Pumpe mit einer maximalen Entnahmekapazität von 1201/s kann hier der Durchfluss reguliert werden. Mittels eines IDM (magnetisch-induktiver Durchflussmesser) ist es möglich, den Durchfluss auf 0,2l/s Genauigkeit zu justieren. Die Länge der Zulaufleitung im Modell beträgt 2,5 m (entspricht $25 \mathrm{~m}$ in der Natur), die Länge des Schotterfangs 1,9m (entspricht $19 \mathrm{~m}$ in der Natur) und die Länge des Ablaufs beträgt $1,3 \mathrm{~m}$ bzw. $13 \mathrm{~m}$ in der Natur (Abb. 2).
Da im Bereich des Krottenbachkanals und des Schotterfangs Freispiegelabfluss vorliegt, wurde das Modellgesetz nach Froude verwendet. Grundlegende Voraussetzung ist hierbei, dass die Froudezahlen in der Natur und im Modell einander entsprechen. Die Froudezahl beschreibt das Verhältnis zwischen Trägheitskräften und Schwerkraft (Kobus 1984).

Als hydraulische Eingangsgrößen wurden Pegeldaten aus der Natur im Zulauf zum Schotterfang verwendet und mit der universellen Fließgleichung (Bollrich 2013) die Durchflussdaten berechnet. Anschließend wurden diese Daten auf die Modellwerte unter Berücksichtigung des Froude'schen Modellgesetzes skaliert. Zur Skalierung der Durchflüsse wurde die Beziehung $\mathrm{Q}_{\mathrm{r}}=\mathrm{L}_{\mathrm{r}}^{5 / 2}$ verwendet. Dabei ist $\mathrm{Q}_{\mathrm{r}}$ die Maßstabszahl des Durchflusses und $L_{r}$ die Längenmaßstabszahl. Zur Untersuchung der hydraulischen Wirksamkeit und der sedimentologischen Rückhaltewirkung im Schotterfang wurden verschiedene Methoden angewendet. Zur qualitativen Untersuchung der Strömung im Schotterfang dienten Farbversuche. Des Weiteren wurde auf Basis einzelner Ereignisse mit erhöhtem Durchfluss bzw. Wasserstand die Auftrittsdauer dieser Ereignisse in der Natur ermittelt, unter Verwendung der Beziehung $\mathrm{t}_{\mathrm{r}}=\mathrm{L}_{\mathrm{r}}{ }^{1 / 2}$ skaliert und als Grundlage für die Verlandungs- bzw. Ausspülversuche mit Sedimenten im Modell verwendet. Dabei entspricht $t_{r}$ der Zeitmaßstabszahl. In Abb. 3 ist ein solches Extremereignis mit einer Dauer von rund einer Stunde bzw. rund $20 \mathrm{~min}$ im Modell exemplarisch dargestellt.

Bei den Verlandungsversuchen wurde jeweils bei geringen Durchflüssen $\left(<0,7 \mathrm{~m}^{3} / \mathrm{s}\right.$ in der Natur bzw. $<21 / \mathrm{s}$ im Modell) Sediment im Zulauf des Schotterfangs zugegeben, um die Absetzwirkung zu untersuchen. Anschließend wurde mit einem Durchfluss von $5 \mathrm{~m}^{3} / \mathrm{s}$ in der Natur (bzw. 16l/s im Modell), angepasst an die Dauer der Abflusswelle in Abb. 3, die sedimentologische Rückhaltewirkung der im Schotterfang angelandeten Sedimente untersucht. Bei den Verlandungs- bzw. Ausspülversuchen basiert die zugegebene Sedimentmenge auf Räumprotokollen des Schotterfanges. Dabei beläuft sich das maximale entnommene Sedimentvolumen auf rund $22 \mathrm{~m}^{3}$ in der Natur bzw. $22 \mathrm{dm}^{3} \mathrm{im}$ Modell. Als Grundlage für die verwendeten Sedimente im Modell dienen die charakteristischen Korngrößen resultierend aus den Siebungen der entnommenen Schotterfangproben. Diese wurden für die Verwendung im Modell im Maßstab 1:10 skaliert.

Zur Analyse der Rückhaltewirkung, definiert als Verhältnis zwischen ausgetragenem Sedimentvolumen bei den Ausspül- bzw. Entlandungsversuchen und zugegebenem Sedimentvolumen bei den Verlandungsversuchen wurde ein Fächerlaser, montiert auf einer automatisierten Messbrücke oberhalb des Modells, verwendet. Der Ablauf zur Aufnahme und zur Berechnung der relevanten Volumina war wie folgt:

1. Vermessung des leeren Schotterfangbeckens (V0).

2. Verlandungsversuch - Sedimentzugabe $\left(22 \mathrm{dm}^{3}\right.$ über $\left.40 \mathrm{~min}\right)$ und Verlandung des Schotterfangs mit $\mathrm{Q}=2 \mathrm{l} / \mathrm{s}$ - Vermessung des Verlandungskörpers im Schotterfang (V1).

3. Entlandungsversuch mit $Q=161 / \mathrm{s}$ (Abb. 3) - Vermessung der Entlandung im Schotterfang (V2).

4. Berechnung der Volumina auf Basis der Laserscans:

a. V1 - V0 = Volumen des Verlandungskörpers $\left(\mathrm{V}_{\mathrm{Ver}}\right)$,

b. V2 - V0 = Volumen nach der Entlandung durch Ausspülung ( $\left.V_{\text {Ent }}\right)$,

c. $V_{\text {Ent }} / V_{\text {Ver }}=$ sedimentologische Rückhaltewirkung $\left(\mathrm{R}_{\mathrm{Sed}}\right)$.

Je näher der Verhältniswert zwischen $V_{\text {Ent }}$ und $V_{\text {Ver }}$ bei Eins liegt, desto besser ist die Rückhaltewirkung hinsichtlich der Sedimente ( $\left.\mathrm{R}_{\text {Sed }}\right)$ im Schotterfang.

\section{Ergebnisse}

\subsection{Ergebnisse Beprobung des natürlichen und urbanen Einzugsgebietes}

In Abb. 4 sind die Ergebnisse der Siebanalyse anhand der charakteristischen Korndurchmesser von $d_{10}$ bis $d_{90}$ als Box-Plot dargestellt, wobei $d_{50}$ dem Median der Probe entspricht (Grenze 


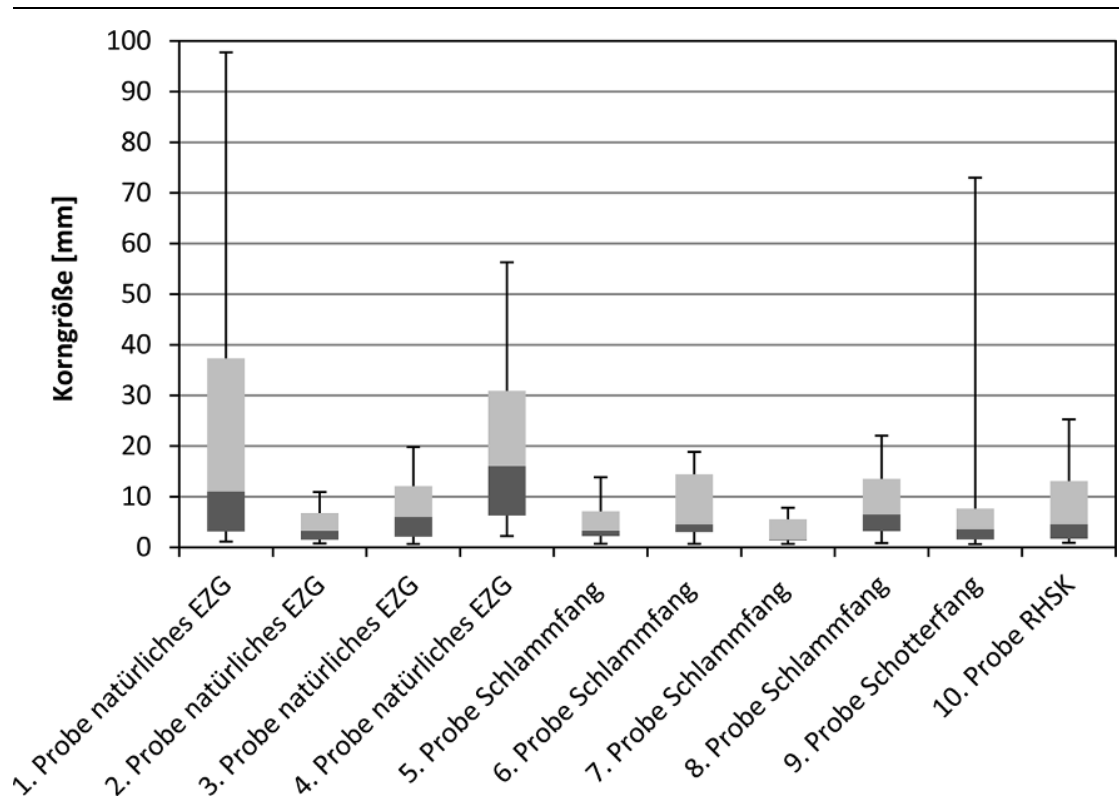

Abb. 4 Korngrößenverteilungen im natürlichen und urbanen Einzugsgebiet sowie im Schotterfang und RHSK. Die Whisker beschreiben die Bereiche der Korngrößen zwischen $\mathrm{d}_{10}$ und $\mathrm{d}_{90}$. Die Box stellt den Bereich zwischen $\mathrm{d}_{25}$ und $\mathrm{d}_{75}$ dar

口Flysch $\square$ Kalk $\square$ Rest

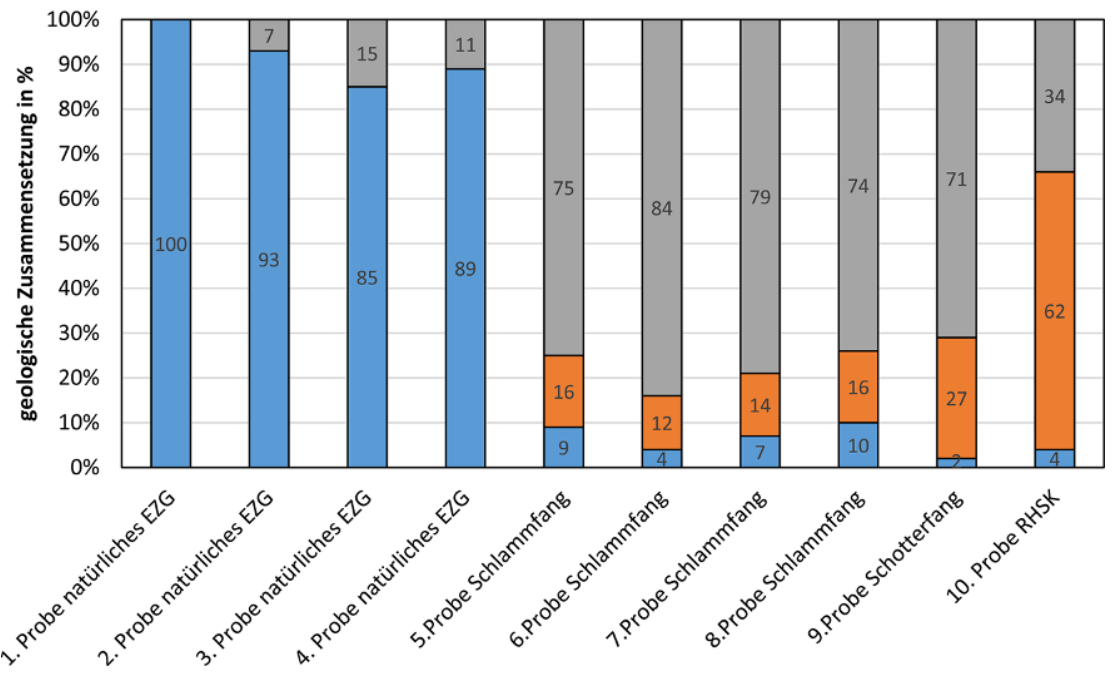

Abb. 5 Geologische Zusammensetzung der Proben im natürlichen und urbanen Einzugsgebiet sowie im Schotterfang und RHSK laut Geröllanalyse

zwischen hell und dunkelgrau). Anhand der Korngrößenverteilung der Proben ist ersichtlich, dass die Verteilungen der Korndurchmesser im natürlichen Einzugsgebiet größere Schwankungen aufweisen als jene im urbanen Einzugsgebiet. Auffällig ist vor allem der Sprung zwischen der ersten und zweiten Probe. Dieser Unterschied ist durch ein Rückhaltebecken, ca. $100 \mathrm{~m}$ flussab der ersten und $10 \mathrm{~m}$ flussab der zweiten Probe bedingt. Während sich im Nahbereich des Rückhaltebeckens feinere den Kornverteilungen im natürlichen Einzugsgebiet weisen die Proben aus dem urbanen Einzugsgebiet eine homogenere Kornverteilung auf. Hier beläuft sich der gemittelte $\mathrm{d}_{50}$ der Proben (5 bis 8 ) auf $3,9 \mathrm{~mm}$ und liegt von der Größenordnung zwischen jenen des Schotterfangs und des RHSK.

Abb. 5 beschreibt die geologische Zusammensetzung der entnommenen Proben. Es ist deutlich erkennbar, dass der Flyschanteil bei den Proben 1 bis 4 im natürlichen Einzugsgebiet, mit Anteilen zwischen 85 bis $100 \%$, dominiert. Neben dem dominanten Flyschanteil konnten im natürlichen Einzugsgebiet Reste, die aus organischem Material, Ziegeln, Tonstücken und Beton bestehen, mit Anteilen zwischen 0 und 15\% festgestellt werden. Der hohe Flyschanteil im natürlichen Einzugsgebiet ist aufgrund der Geologie in diesem Bereich zu erwarten. Die Proben 5 bis 8 wurden aus den Schlammfängen entnommen und weichen von der geologischen Zusammensetzung deutlich von jenen aus dem natürlichen Einzugsgebiet ab. Es ist erkennbar, dass hier der Restanteil mit bis zu $84 \%$ dominiert. Der Flyschanteil ist mit 4 bis $10 \%$ deutlich geringer als bei den Proben 1 bis 4. Neu hinzu kommt der Kalkanteil, der zwischen 12 und $16 \%$ liegt. Die Proben aus dem Schotterfang und dem RHSK weisen eine ähnliche geologische $\mathrm{Zu}$ sammensetzung wie die Proben 5 bis 8 auf. Im Schotterfang beläuft sich der Flyschanteil auf $2 \%$, der Kalkanteil auf $27 \%$ und der Restanteil auf $71 \%$. Im RHSK ist der Flyschanteil mit $4 \%$, wie schon im Schotterfang, am geringsten. Dafür ist hier der Kalkanteil mit $62 \%$ dominierend.

\subsection{Ergebnisse direkte Geschiebemessung}

In Tab. 1 beschreibt Q den Durchfluss des Erbsenbaches in $\mathrm{m}^{3} / \mathrm{s}, \mathrm{S}$ die gemessene absolute Sedimentmenge in $\mathrm{kg}$ und $\mathrm{q}_{\mathrm{s}}$ die Geschiebemenge pro Stunde $\mathrm{h}$ und Breitenmeter $\mathrm{m}$. Ml bis M3 wurde mittels Helley-Smith Sampler beprobt und M4 bis M6 mit dem Handfänger. Für M1 bis M6 wurde die Messdauer auf $30 \mathrm{~min}$ festgelegt. M7 und M8 beschreiben Langzeitmessungen mittels Geschiebekorb. Der Durchfluss während der Messung M5 ist mit $0,05 \mathrm{~m}^{3} / \mathrm{s}$ nur geringfügig höher als der mittlere Durchfluss des Erbsenbaches $\left(0,04 \mathrm{~m}^{3} / \mathrm{s}\right)$. Bei den Messungen M1 bis M4 und M6 bis M7 wurde bei erhöhten 
Tab. 1 Ergebnisse der Geschiebemessung. Dabei steht $Q$ für Durchfluss in $\mathrm{m}^{3} / \mathrm{s}, \mathrm{S}$ für absolute gefangene Sedimentmenge in $\mathrm{kg}$ bezogen auf die Messdauer und q für Sedimenttransportrate in $\mathrm{kg} / \mathrm{hm}$

\begin{tabular}{|l|l|l|l|l|}
\hline Geschiebe-Messungen & Messdauer & $Q\left(\mathrm{~m}^{3} / \mathrm{s}\right)$ & \multicolumn{2}{c}{$\mathrm{S}(\mathrm{kg})$} \\
\hline M1 bis M3 & $30 \mathrm{~min}$ & 0,118 & 0,02 & 0,79 \\
\hline M4 & $30 \mathrm{~min}$ & 0,089 & 0,002 & 0,008 \\
\hline M5 & $30 \mathrm{~min}$ & 0,046 & 0,04 & 0,28 \\
\hline M6 & $30 \mathrm{~min}$ & 0,066 & 0,1 & 0,832 \\
\hline M7 & 16 Tage & 0,276 (Maximal) & 0,6 & 0,004 \\
\hline M8 & 45 Tage & 0,065 (Maximal) & 1,4 & 0,003 \\
\hline
\end{tabular}

Abflüssen im Vergleich zum mittleren jährlichen Durchfluss gemessen. Dennoch deuten die Ergebnisse auf nur geringen Geschiebetransport hin. Bei einem Durchfluss von $0,118 \mathrm{~m}^{3} / \mathrm{s}$ beträgt die gemessene Sedimentmenge $S$ lediglich $0,02 \mathrm{~kg}$ über einen Messzeitraum von $30 \mathrm{~min}$. Die größte aufgenommene Transportrate $\mathrm{q}_{\mathrm{s}}$ wurde bei einem Durchfluss von $0,066 \mathrm{~m}^{3} / \mathrm{s}$ gemessen und beträgt $0,832 \mathrm{~kg} / \mathrm{hm}$. Auch bei den Langzeitmessungen konnte kaum Sedimenttransport gemessen werden. Über einen Zeitraum von 61 Tagen (M7 und M8) beläuft sich die gemessene Sedimentmenge auf $2,0 \mathrm{~kg}$, trotz einer Durchflussspitze von $0,276 \mathrm{~m}^{3} / \mathrm{s}$. Tab. 1 fasst die Ergebnisse der Geschiebemessungen zusammen. Bei den Langzeitmessungen M7 und M8 wurde jeweils der maximale Durchfluss während des Messzeitraums angegeben.

Die Messungen zeigen, dass die Geschieberückhaltemaßnahmen im Einzugsgebiet für die gemessenen Ereignisse funktionieren, was die geologische Untersuchung mit dem Ergebnis des Fehlens von Flyschanteilen im Schotterfang bestätigt. Aussagen über den Sedimenttransport bei Extremereignissen, wie z.B. einem $\mathrm{HQ}_{1}$, können anhand der Messungen nicht getroffen werden, da während der Messperiode kein Extremereignis auftrat.
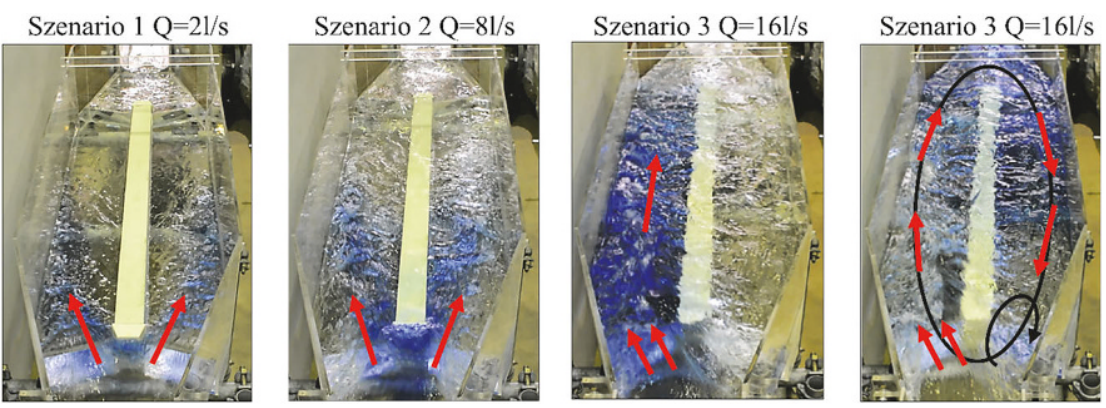

Abb. 6 Farbversuche bei Durchflüssen von $2 \mathrm{l} / \mathrm{s}, 8 \mathrm{l} / \mathrm{s}$ und 16l/s zur qualitativen Bewertung der Strömung

\subsection{Ergebnisse physikalisches Modell und Optimierung}

\subsubsection{Ist-Stand}

Um die hydraulische Wirksamkeit zu untersuchen, wurde der Durchfluss im Schotterfang schrittweise gesteigert, um die Strömungszustände bei verschiedenen Durchflüssen zu erfassen. Dabei entsprechen 21/s im Modell $0,63 \mathrm{~m}^{3} / \mathrm{s}$ in der Natur, $81 / \mathrm{s}$ im Modell $2,5 \mathrm{~m}^{3} / \mathrm{s}$ und $16 \mathrm{l} / \mathrm{s}$ im Modell $5,0 \mathrm{~m}^{3} / \mathrm{s}$. Alle gewählten Durchflüsse entsprechen erhöhten Abflüssen im Krottenbachkanal, der einen Trockenwetterabfluss von circa $0,15 \mathrm{~m}^{3} / \mathrm{s}$ besitzt. Um einen qualitativen Überblick über die Strömung im Schotterfang zu gewährleisten, wurden Farbversuche wie in Abb. 6 bei den verschiedenen Durchflüssen, gegliedert in Szenario 1 bis 3, durchgeführt.

Anhand der Farbversuche ist deutlich erkennbar, dass die Strömung beide Schotterfangbecken bis $\mathrm{zu}$ einem Durchfluss kleiner als 161/s gleichmäßig durchströmt. Ab 16l/s (Szenario 3) kommt es zur einseitigen Durchströmung des Schotterfanges und zur Ausbildung einer Kehrströmung. Dieser Effekt tritt zufällig auf und kann von einer Seite auf die andere wechseln. Dadurch erhöhen sich die Fließgeschwindigkeiten im durchströmten
Becken, da nahezu der gesamte Durchfluss durch ein Becken (halber Querschnitt) abgeführt wird. Physikalisch entsteht dieser Effekt bei ,großen Aufweitungen" wie es beim Übergang zwischen Kanal zu Schotterfang der Fall ist. Die Hauptaufgabe der Aufweitung im Schotterfang ist die Reduzierung der Fließgeschwindigkeit sowie die Erzeugung eines einheitlichen Geschwindigkeitsprofils nach der Aufweitung, damit Sedimente abgesetzt werden können. Beim Anstieg des Durchflusses und somit der Fließgeschwindigkeit im Aufweitungsbereich kann die Strömung der Aufweitung nicht mehr folgen und es kommt zu Ablösungen an den Seitenwänden, Rückströmungen (Abb. 7 rechtes Bild mit 161/s) und instationären Phänomenen, die sowohl zu Verlusten als auch zu ungleichmäßigen Abströmverhältnissen führen. Da die Strömung im Bereich der Wände eine geringere kinetische Energie besitzt als im mittleren stark durchströmten Bereich (hohe Fließgeschwindigkeiten), werden die Fließgeschwindigkeiten bei gleichmäßigem Druckanstieg an der Wand früher Null oder negativ und führen zu Asymmetrien im System. Basierend auf kleinen, stets vorhandenen Asymmetrien geschieht dies an einer Seite der Aufweitung früher als an der anderen (Mohringer 2012). Aufgrund dessen kommt es über die Breite $\mathrm{zu}$

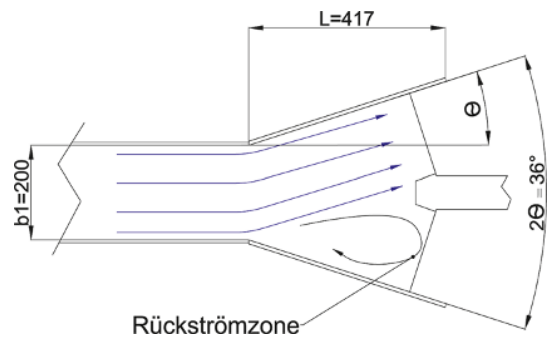

Abb. 7 Strömungssituation an der Aufweitung im Schotterfangmodell und schematische Darstellung der Rückströmzone (Modellmaße in mm) 


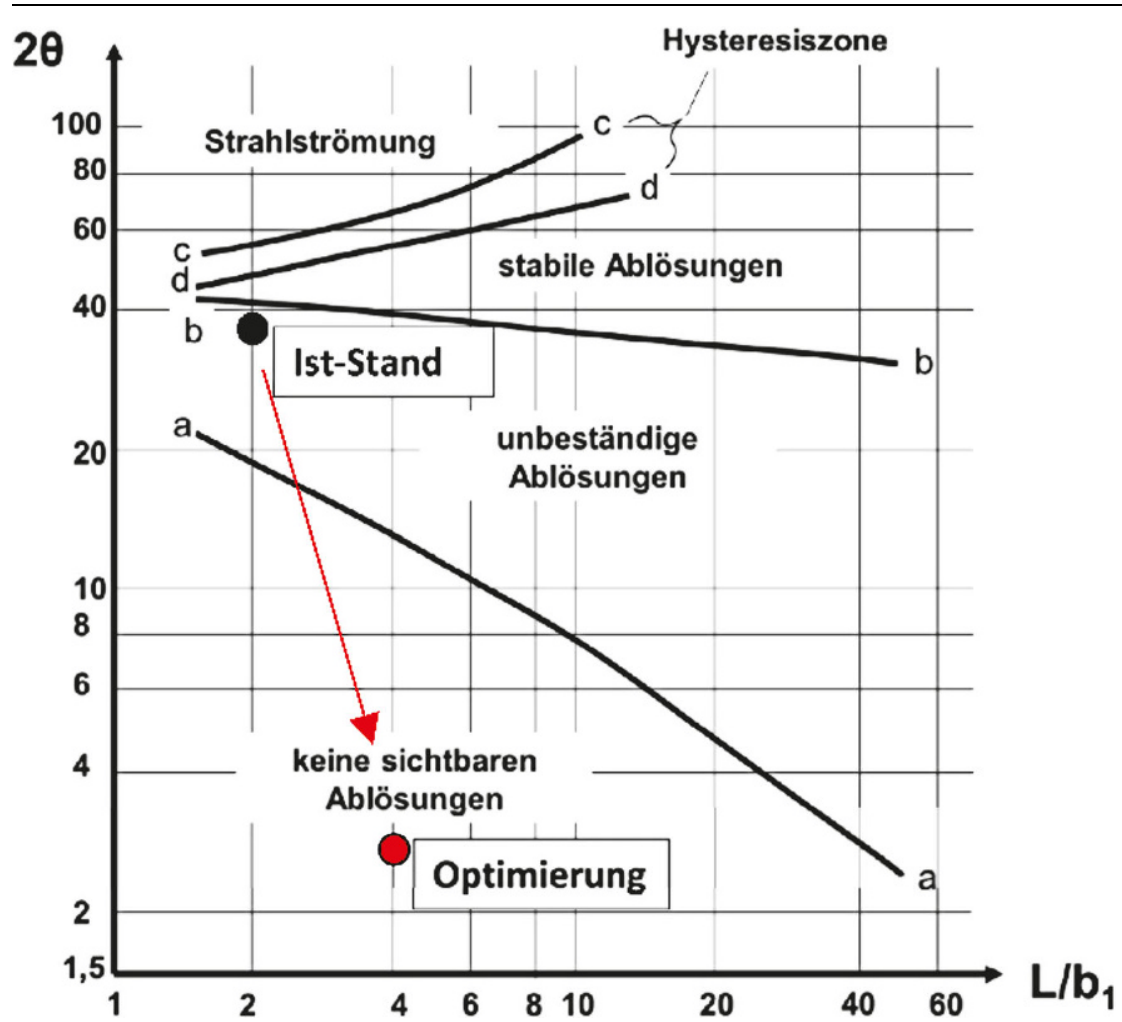

Abb. 8 Ablösezustände der Strömung in Abhängigkeit vom Öffnungswinkel und dem Längen-zu-Breiten-Verhältnis. Der schwarze Punkt beschreibt den Ist-Stand und der rote Punkt den Zustand nach der Optimierung (Renau et al. 1967)

einem asymmetrischen Druckgradienten und die Strömung legt sich an der Seite mit geringerem Druck an. An der anderen Seite entstehen Ablösezonen (Abb. 7), die einen Teil des Querschnitts blockieren. In Anlehnung an den beobachteten instationären Vorgang kann dieser mit den von Kline (1959) defi-
Wie Abb. 7 veranschaulicht, besitzt die Aufweitung des Schotterfangs im Modell einen Öffnungswinkel $2 \Theta=36^{\circ}$, eine Länge $\mathrm{L}=417 \mathrm{~mm}$ und eine Eingangsbreite $\mathrm{bl}=200 \mathrm{~mm}$. Das Verhältnis von L/b1 beträgt ca. 2,1 und bei vorhandenem Öffnungswinkel ergibt sich laut dem Diagramm nach Renau et al. (1967) der Zustand im Übergangsbereich zur stabilen Ablösung (Abb. 8). Dies deckt sich mit den Beobachtungen der Farbversuche.

Um den Einfluss der asymmetrischen Strömung auf die sedimentologische Rückhaltewirkung zu beziffern, wurden Ausspülversuche des Schotterfangs durchgeführt (die folgenden Größen sind in Modellmaßen angegeben). Es wurden $22 \mathrm{dm}^{3}$ Sediment mit Korngrößen zwischen 0,4 bis $0,8 \mathrm{~mm}$ (das entspricht $\mathrm{d}_{50}$ bis $\mathrm{d}_{90}$ des Schotterfangs), bei einem Durchfluss $Q=21 / s$ im Zulauf zum Schotterfang zugegeben. Die Sedimentmenge entspricht dabei ca. $40 \%$ des maximalen Rückhaltevermögens des Schotterfangs. Abb. 9 zeigt die schrittweise Verlandung und Entlandung des Schotterfanges.

Nachdem das gesamte Sediment zugegeben war $(40 \mathrm{~min})$, wurde der Durchfluss auf 161/s erhöht, $20 \mathrm{~min}$ konstant gehalten und danach wieder reduziert. Dies entspricht in etwa dem skalierten Zeitraum des Extremereignisses, wie in Abb. 3 dargestellt. Anhand der Sedimentversuche ist ersichtlich, dass es zur Bildung einer ausgeprägten Sedimentfront kommt, die nach $3 \mathrm{~min}$ nahezu das hintere Ende des Schotterfangs erreicht und so Sedimentaustrag aus dem Schotterfangbecken verur-
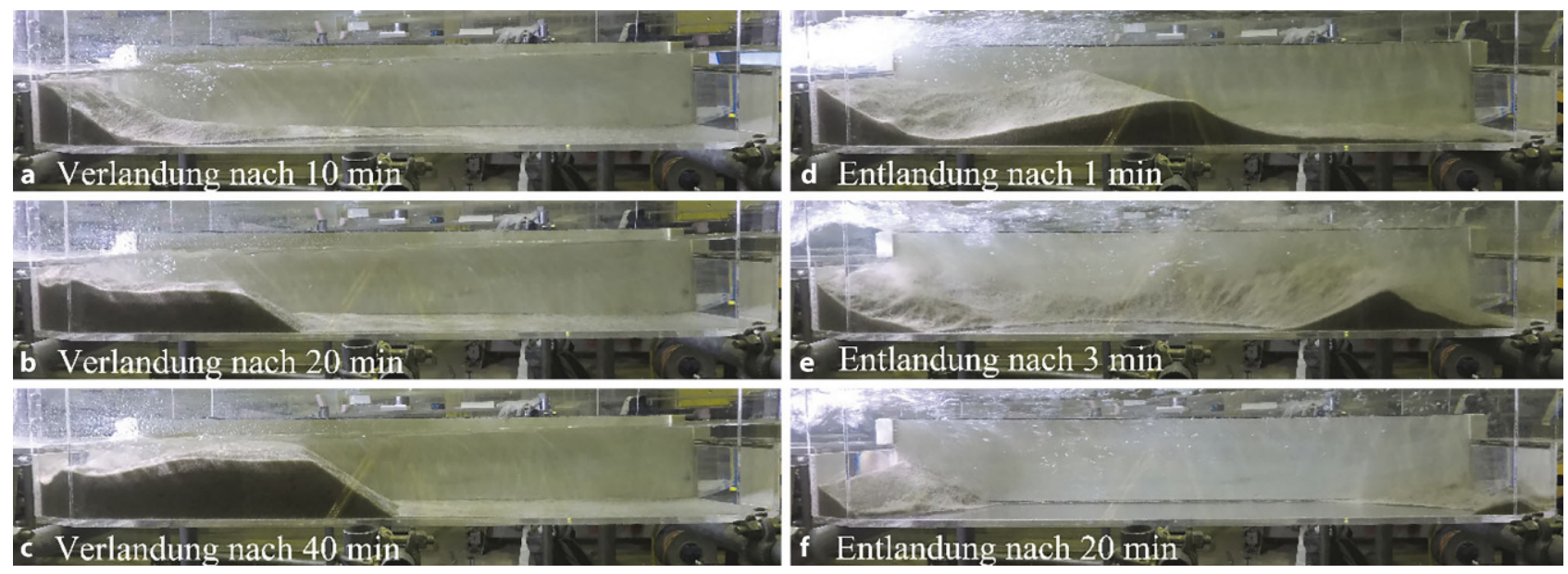

Abb. 9 Ver- und Entlandung des Schotterfangs für den Ist-Stand. Die Verlandungsversuche $\mathbf{a}, \mathbf{b}$ und $\mathbf{c}$ wurden bei einem Durchfluss $Q=21 / s$ durchgeführt. Das zugegebene Sedimentvolumen beläuft sich dabei auf $22 \mathrm{dm}^{3}$ in $40 \mathrm{~min}$. Der Durchfluss bei Entlandungsversuchen d, e und $\mathbf{f}$ beträgt $16 \mathrm{l} / \mathrm{s}$ und wurde 20 min konstant gehalten. Fließrichtung von links nach rechts 


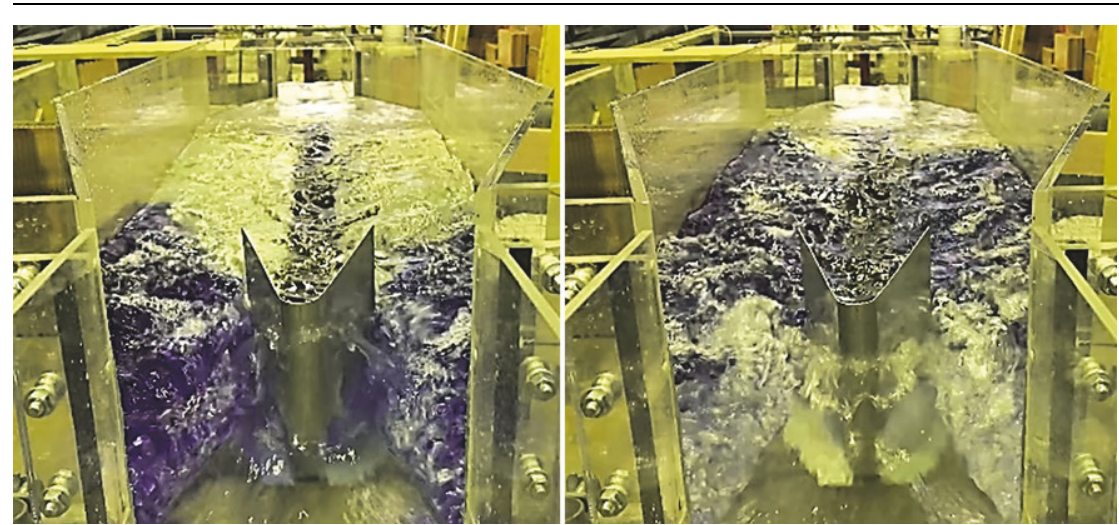

Abb. 10 Farbversuche mit Strömungsbrecher bei einem Durchfluss $Q=16 \mathrm{l} / \mathrm{s}$

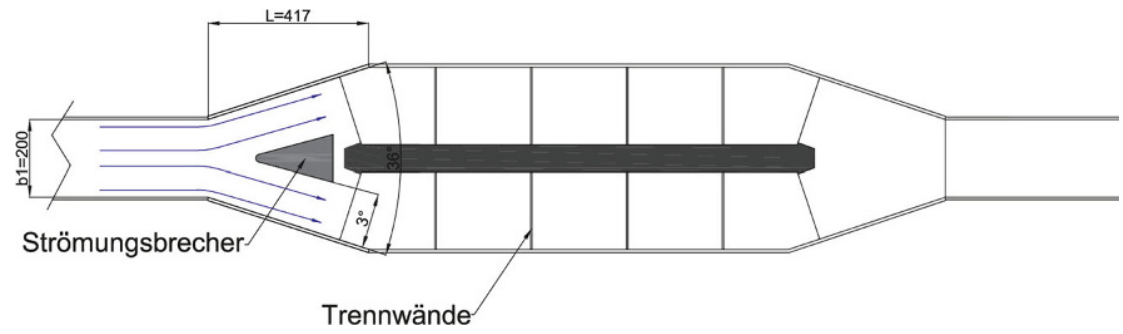

Abb. 11 Schematische Darstellung des optimierten Schotterfangs im physikalischen Modell, inklusive Trennwände und Strömungsbrecher (Modellmaße in mm)

sacht. Anhand der Fächerlaserscans wurden die relevanten Volumina berechnet. Das verlandete Volumen $\left(\mathrm{V}_{\mathrm{Ver}}\right)$ im aufgenommenen Schotterfangbecken beläuft sich auf $11 \mathrm{dm}^{3}$, wodurch von einer symmetrischen Sedimentverlandung hinsichtlich der beiden Schotterfangbecken ausgegangen werden kann. Nach der Erhöhung des Durchflusses (Entlandungsversuch) wurde ein Restvolumen von $2 \mathrm{dm}^{3}$ ( $\mathrm{V}_{\text {Ent }}$ ) gemes- sen. Daraus ergibt sich eine Differenz von $9 \mathrm{dm}^{3}$ zwischen $V_{\text {Ver }}$ und $V_{\text {Ent }}$ und eine Rückhaltewirkung $R_{\text {Sed }}$ von 0,18 bzw. von $18 \%$ im gemessenen Schotterfangbecken.

Dies entspricht in Naturwerten rund $9 \mathrm{~m}^{3}$ die aus dem Schotterfang bei einem Extremereignis ausgetragen werden. Während das stark durchströmte Becken, hier in Fließrichtung links (Abb. 9), nahezu komplett ausgespült wird, kommt es im rechten Becken auf Basis optischer Kontrolle nur $\mathrm{zu}$ sehr geringem Sedimentaustrag.

\subsubsection{Optimierung}

Um der beschriebenen Ausspülungstendenz aufgrund der hydraulisch einseitigen Durchströmung entgegenzuwirken, wurde der Schotterfang im physikalischen Modell optimiert. Dafür wurde ein keilförmiger Strömungsbrecher, dessen Winkel $3^{\circ}$ vom Öffnungswinkel des Schotterfanges abweicht, im Bereich der Aufweitung eingebaut (die folgenden Größen sind in Modellmaßen angegeben): Der Strömungsbrecher hat eine Länge von $200 \mathrm{~mm}$ und eine Höhe von $170 \mathrm{~mm}$. Durch die Reduktion des Öffnungswinkels von $36^{\circ}$ auf $3^{\circ}$ ist nach Abb. 8 nicht mit Ablösungserscheinungen zu rechnen. Dies konnte mit Farbversuchen bei einem Durchfluss von 161/s bestätigt werden (Abb. 10).

$\mathrm{Da}$ es zur Bildung einer ausgeprägten Sedimentfront und dadurch zum Austrag des Sediments kommt, wurden Trennwände quer zur Fließrichtung in die Schotterfangbecken, über die gesamte Breite, eingebaut. Insgesamt wurden pro Becken vier Trennwände mit einer Höhe von $60 \mathrm{~mm}$ (halbe Beckentiefe) und symmetrischen Abständen von jeweils $240 \mathrm{~mm}$ angeordnet. Diese wirken wie Kammern und sollen durch kontinuierliches Auffüllen mit Sedimenten die Ausbildung einer dominanten Sedimentfront verhindern und die Geschwindigkeit der abgetragenen bzw. abgeschwächten Sediment-
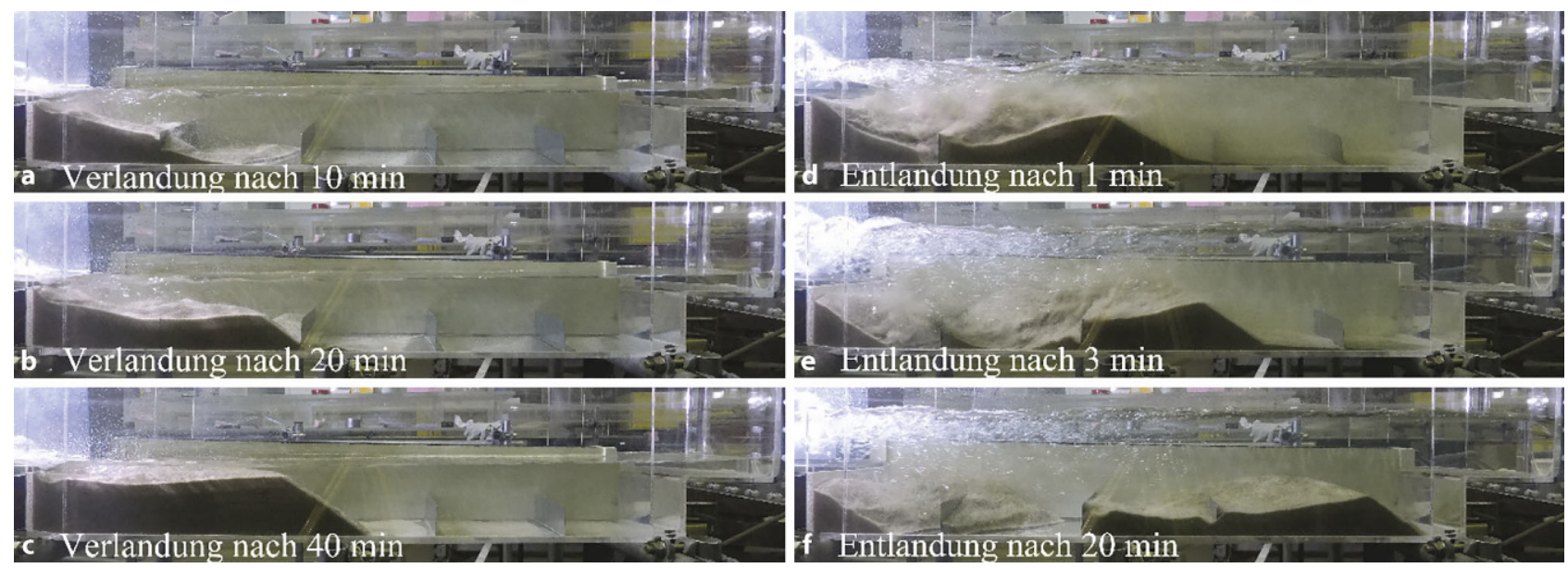

Abb. 12 Ver- und Entlandung des Schotterfanges nach der Optimierung. Die Verlandungsversuche $\mathbf{a}, \mathbf{b}$ und $\mathbf{c}$ wurden bei einem Durchfluss Q = 21/s durchgeführt. Das zugegebene Sedimentvolumen beläuft sich dabei auf $22 \mathrm{dm}^{3}$ in $40 \mathrm{~min}$. Der Durchfluss bei Entlandungsversuchen $\mathbf{d}$, e und $\mathbf{f}$ beträgt $16 \mathrm{l} / \mathrm{s}$ und wurde 20 min konstant gehalten. Fließrichtung von links nach rechts 


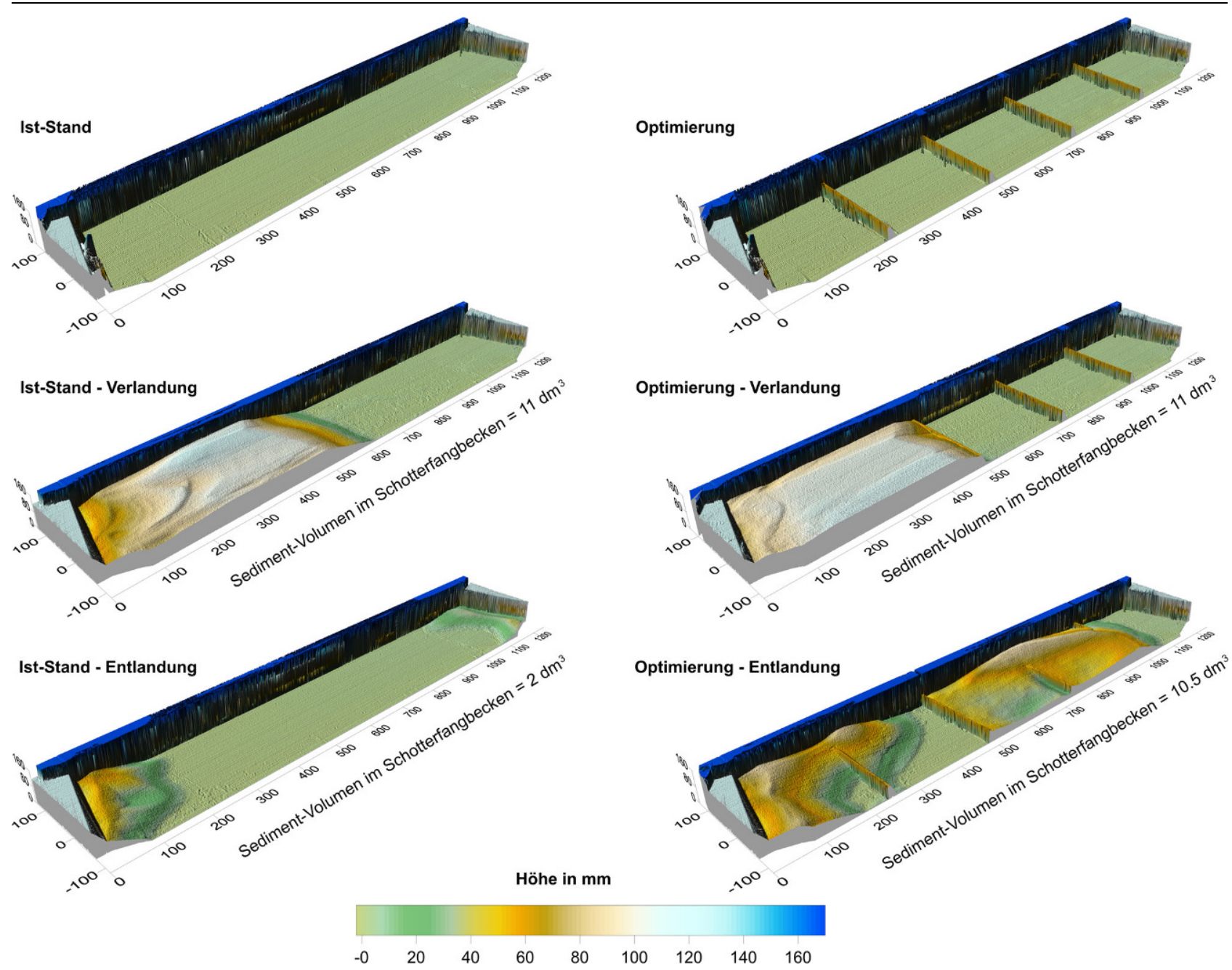

Abb. 13 Vergleich der Verlandung und Entlandung des Schotterfangs zwischen dem Ist-Stand (a) und der Optimierungsvariante (b)

front verlangsamen. Abb. 11 stellt die Optimierungsvariante dar.

Durch den Strömungsbrecher und die Anordnung der Trennwände konnte bei gleicher Sedimentzugabe und hydraulischen Eingangsgrößen, wie bei der Variante des Ist-Stands, vor allem hinsichtlich der Entlandung ein besseres Ergebnis erzielt werden. Während die Verlandung ähnlich verläuft, ist in Abb. 12 gut erkennbar, dass durch die Trennwände und den Strömungsbrecher die Ausbildung der Sedimentfront sowie die Geschwindigkeit der Sedimentbewegung reduziert werden konnten.

Basierend auf den Laserscandaten konnte festgestellt werden, dass es bei der Optimierungsvariante zu minimalem Austrag kommt. So beläuft sich das ausgetragene Volumen $\left(\mathrm{V}_{\text {Ver }}-\mathrm{V}_{\mathrm{ent}}\right)$ in einem Schotterfangbecken auf $0,5 \mathrm{dm}^{3}$ und die sedimentologische Rückhalte- wirkung auf 0,95 bzw. auf $95 \%$. Abb. 13 stellt die Verlandung sowie den Zustand nach dem simulierten Extremereignis vergleichend zwischen dem Ist-Stand und der Optimierung für ein Schotterfangbecken dar. Fließrichtung von links nach rechts.

Nachdem der Verlandungs- sowie der Entlandungszustand nach den Experimenten symmetrisch war, kann bei der Optimierungsvariante insgesamt von rund $1 \mathrm{dm}^{3}$ Sedimentaustrag ausgegangen werden, das entspricht in Naturmaßen $1 \mathrm{~m}^{3}$.

\section{Zusammenfassung und Schlussfolgerung}

Diese Arbeit beschäftigt sich mit dem Sedimenteintrag und Sedimentverlandungen in Abschnitten der Wiener Kanalisation (1190 Wien). Im urbanen Bereich gibt es oft eine Vielzahl an
Möglichkeiten, über die Sedimente in das Kanalsystem eingetragen werden können, wie zum Beispiel über Straßeneinläufe oder über Bäche, die in das Kanalsystem einmünden. Im betrachteten Projektgebiet kommt es im rechten Hauptsammelkanal (RHSK) im Bereich der Heiligenstädter Straße zu Sedimentverlandungen, die in diesem Abschnitt händisch geräumt werden müssen. Dem RHSK ist ein Schotterfang vorgelagert, in dem Sediment aus dem urbanen Raum und den Wienwaldbächen (Krottenbach und Erbsenbach) gesammelt werden soll. Vor allem bei erhöhten Abflüssen ist die Rückhaltewirkung des Schotterfangs nur teilweise gegeben und es kommt zu Sedimentaustrag in den RHSK. Ziel dieser Arbeit ist es, die Sedimentherkunft der im RHSK verlandeten Sedimente zu bestimmen und die hydraulische sowie die sedimentologische Rückhaltewir- 
kung des Schotterfangs zu untersuchen und $\mathrm{zu}$ optimieren. Zur Umsetzung der Ziele wurden Siebanalysen, Geröllanalysen und Geschiebemessungen durchgeführt sowie der Schotterfang im physikalischen Modell untersucht und optimiert.

Aufgrund der durchgeführten Siebanalyse und der daraus resultierenden charakteristischen Korngrößen ist die Übereinstimmung zwischen den Proben aus dem urbanen Einzugsgebiet mit den Proben aus dem Schotterfang und dem RHSK größer als mit den Proben aus dem natürlichen Einzugsgebiet. Ein ähnliches Ergebnis liefert die geologische Untersuchung (Geröllanalyse). Die Ergebnisse der Geröllanalyse weisen darauf hin, dass das verlandete Sediment im Schotterfang bzw. im RHSK nur zu sehr geringen Anteilen ( 2 bis $4 \%$ ) aus dem natürlichen Einzugsgebiet stammt. Nachdem Kalkanteile nur in den Proben aus dem urbanen Einzugsgebiet (Probe 5 bis 8 aus den Schlammfängen) und dem Schotterfang sowie dem RHSK vorkommen, ist der Eintrag der Sedimente aus dem urbanen Einzugsgebiet bzw. über die Straßeneinläufe wahrscheinlich. Dieses Ergebnis kann auch aus der Geschiebemessung am Erbsenbach abgeleitet werden. Hier konnte trotz höherer Abflüsse im Vergleich zum mittleren jährlichen Abfluss $\left(0,04 \mathrm{~m}^{3} / \mathrm{s}\right)$ bis $\mathrm{zu} 0,27 \mathrm{~m}^{3} / \mathrm{s}$ kaum Sedimenttransport festgestellt werden. Auch die Langzeitmessungen deuten auf wenig Geschiebeführung im Erbsenbach und somit auf kaum Sedimenteintrag aus dem natürlichen Einzugsgebiet in die Kanalisation hin. Generell scheinen damit die Geschieberückhaltemaßnahmen im Einzugsgebiet gut $\mathrm{zu}$ funktionieren. Hingegen gibt es aus dem urbanen Einzugsgebiet Sedimenteintrag, woraus sich ein Handlungsbedarf ableitet, denn prinzipiell und primär sollte der Eintrag in das Kanalsystem verringert werden.

Basierend auf der hydraulischen sowie der sedimentologischen Untersuchung des Schotterfangs konnte festgestellt werden, dass es aufgrund der Aufweitungsgeometrie am Beginn des
Schotterfangs zu einer asymmetrischen Anströmung bei erhöhten Durchflüssen $\left(>5 \mathrm{~m}^{3} / \mathrm{s}\right.$ in der Natur) kommt. Diese verursacht eine erhöhte Fließgeschwindigkeit in einem der beiden Schotterfangbecken, während die Rückströmung im anderen Schotterfangbecken als Blockade für den Abfluss wirkt. Anhand von Verlandungs- und Entlandungsversuchen konnte festgestellt werden, dass $18 \%$ der verlandeten Sedimente nach einem Extremereignis im Schotterfang verweilen. Der Rest, rund $9 \mathrm{~m}^{3}$, wird ausgespült. Zur Verbesserung der hydraulischen Situation und in weiterer Folge auch der sedimentologischen Rückhaltewirkung wurde der Schotterfang im physikalischen Modell durch den Einbau eines Strömungsbrechers im Zulaufbereich (Aufweitungsbereich) sowie durch Trennwände in den Schotterfangbecken optimiert. Durch die Optimierung konnte die asymmetrische Anströmung verhindert werden, und die Trennwände reduzierten die Transportgeschwindigkeit der Sedimente, wodurch diese bei dem simulierten Extremereignis im Schotterfang verweilen. Auf Basis der Optimierung konnte die Rückhaltewirkung im Vergleich zum Ist-Stand von 18 auf $95 \%$ erhöht werden.

Basierend auf den Untersuchungsergebnissen stammen die Sedimente im RHSK aus dem urbanen Raum des Projektgebiets. Diese verlanden aufgrund der Ausspültendenz des Schotterfangs bei erhöhten Abflüssen im RHSK, was großen Instandhaltungsaufwand verursacht.

Zusammenfassend ist festzuhalten, dass die gesamtheitliche Betrachtung Untersuchung der natürlichen und urbanen Einzugsgebiete, Geschiebemessungen und physikalische Betrachtung des Schotterfanges im Modell - bei der Auffindung der Problemquelle hinsichtlich der Verlandungserscheinungen gut geeignet war. Dadurch können sowohl Verbesserungsmaßnahmen am Schotterfang durchgeführt werden als auch die konzeptionelle Vorgehensweise der hier vorgestellten Methoden auf andere Stellen mit Verlandungsproblemen in der Kanalisation angewendet werden.

\section{Ausblick}

Neben den bereits durchgeführten Verlandungs- und Entlandungsversuchen, ausgelegt auf die Dauer von kurzen Starkregenereignissen, sollen auch Versuche mit einer längeren Versuchszeit durchgeführt werden, um gegebenenfalls den aktuellen Optimierungsstand weiter $\mathrm{zu}$ verbessern. Um die Umsetzung in die Natur möglichst materialund kosteneffizient zu gestalten, sind Detailoptimierungen am Strömungsbrecher vorgesehen. Die Auswirkungen der Detailoptimierungen auf die hydraulische Situation (Fließgeschwindigkeiten, Turbulenzen, hydraulische Instabilitäten) im Schotterfang soll mittels „Particle Image Velocimetry“ (PIV) aufgenommen und mit Ver- und Entlandungsversuchen verglichen werden. Auch gibt es Überlegungen, die Wirksamkeit von Schwallspülungen zur Verringerung der Ablagerungen im rechten Hauptsammelkanal zu untersuchen.

Danksagung Die AutorInnen bedanken sich bei Wien Kanal für die Finanzierung des Forschungsprojekts sowie für die Bereitstellung von Daten. Der Dank der AutorInnen gilt Thomas Gold, Kevin Reiterer, Josef Pölzl, Johannes Fischer, Dominik Worf, Wolfgang Summer und Franz Ottner für ihre Unterstützung.

Funding Open access funding provided by University of Natural Resources and Life Sciences Vienna (BOKU).

Open Access Dieser Artikel wird unter der Creative Commons Namensnennung 4.0 International Lizenz (http:// creativecommons.org/licenses/by/4. $0 /$ deed.de) veröffentlicht, welche die Nutzung, Vervielfältigung, Bearbeitung, Verbreitung und Wiedergabe in jeglichem Medium und Format erlaubt, sofern Sie den/die ursprünglichen $\mathrm{Au}$ tor(en) und die Quelle ordnungsgemäß nennen, einen Link zur Creative Commons Lizenz beifügen und angeben, ob Änderungen vorgenommen wurden. 


\section{Originalarbeit}

\section{Literatur}

Bollrich, G. (2013): Technische Hydromechanik 1 - Grundlagen. 7. Auflage. Berlin: Beuth Verlag $\mathrm{GmbH}$.

Bundesministerium für Land- und Forstwirtschaft, Umwelt und Wasserwirtschaft (2014): Digitale Gewässerkartei. Wien: Bundesministerium für Land- und Forstwirtschaft, Umwelt und Wasserwirtschaft.

Kline, S. J, Abbott, D.E. und Fox, R.W. (1959): Optimum Design of Straight-Walled Diffusers. Journal of Basic Engineering. 81, 321-331.

Kobus, H. (1984): Wasserbauliches Versuchswesen. 2. Auflage. Hamburg: Schriftenreihe des
Deutschen Verbandes für Wasserwirtschaft und Kulturbau. Verlag Paul Parey.

Mohringer, T. (2012): Entwurf von Ein- und Auslaufbauwerken von Pumpspeicherwerken. Dissertation. Fakultät für Bauingenieur- Geo- und Umweltwissenschaften des Karlsruher Instituts für Technologie.

Renau, L. R., Johnston, J. P. und Kline, S. J. (1967): Performance and Design of Straight, Two-Dimensional Diffusers. Journal of Basic Engineering 3(1), 141-150. https://doi.org/10. $1115 / 1.3609544$.
Schnabel, W. (2002): Geologische Karte von Niederösterreich 1:200.000. Wien: Geologische Bundesanstalt.

Wien Kanal (2016): Kanalinformationssystem Kanal mit MZK, Wien (http://www.kanis.at/).

Wien Kanal (2017): Leistungsbericht der Unternehmung Wien Kanal, Wien (https://www.wien. gv.at/statistik/leistungsbericht/wienkanal/index. html) 